\title{
A mathematical model for Ebola epidemic with self-protection measures
}

\author{
T. Berge ${ }^{\dagger \ddagger}, \quad$ M. Chapwanya ${ }^{\ddagger}$, J. M. -S. Lubuma ${ }^{\ddagger *}$ and Y. A. Terefe T $^{\ddagger}$ \\ $\dagger$ Department of Mathematics and Computer Sciences, University of Dschang, Cameroon \\ ${ }^{\ddagger}$ Department of Mathematics and Applied Mathematics, University of Pretoria 0002, South \\ Africa \\ berge.tsanou@up.ac.za,m.chapwanya@up.ac.za, \\ jean.lubuma@up.ac.za, yibeltal.terefe@up.ac.za
}

\begin{abstract}
A mathematical model presented in Ref. 7 for the transmission dynamics of Ebola virus is extended to incorporate vaccination and change of behavior for self-protection of susceptible individuals. In the new setting, it is shown that the disease-free equilibrium is globally asymptotically stable when the basic reproduction number $\mathcal{R}_{0}$ is less than or equal to unity and unstable when $\mathcal{R}_{0}>1$. In the latter case, the model system admits at least one endemic equilibrium point, which is locally asymptotically stable. Using the parameters relevant to the transmission dynamics of the Ebola virus disease, we give sensitivity analysis of the model. We show that the number of infectious individuals is much smaller than that obtained in the absence of any intervention. In the case of the mass action formulation with vaccination and education, we establish that the number of infectious individuals decreases as the intervention efforts increase. In the new formulation, apart from supporting the theory, numerical simulations of a nonstandard finite difference scheme that we have constructed suggests that the results on the decrease of the number of infectious individuals is valid.
\end{abstract}

Keywords: Ebola virus disease; dynamical systems; behaviour change; sensitivity analysis; nonstandard finite difference method.

\section{Introduction}

The Ebola virus disease (EVD) is named so after the Ebola River valley in Zaire (now Democratic Republic of Congo) where it appeared for the first time in 1976 (Ref. 35). Since then, other outbreaks have been identified in parts of Central Africa, see for example Refs. 1,7,8,13,35. The most devastating outbreak was observed recently (2014) in some West African countries: Guinea, Liberia, Nigeria, Senegal and Sierra Leone. In almost all the outbreaks, the initial infection is due to contacts with infected animals (hunted for food), such as fruit bats and primates (ape, monkey, chimpazee) (Refs. 7,22,23). This highlights the importance of considering the indirect contacts with the surrounding environment as a transmission route of the virus.

${ }^{*}$ Corresponding Author 
Of course, the direct transmission of EVD cannot be underestimated. Typically, the direct transmission involves contact with blood or body fluid and with objects that have been contaminated with Ebola viruses. A detailed literature review on direct and indirect transmission of EVD is given in Ref. 7 and the references therein.

The model investigated in Ref. 7 enriched the few existing models by incorporating the indirect transmission route in the following specific manner:

(i) Infection through the contaminated environment resulting from African practices, hospitality and poor hygienic conditions;

(ii) Provision or recruitment source of Ebola virus linked to the consumption of bats, hunted meat and fruits from the rain-forest.

The findings in Ref. 7 with regard to the control strategy of EVD included educating the population against the practices contained in items (i) and (ii) above. In the present paper, we broaden the education of the population in two additional ways outlined below.

(iii) We add a vaccinated compartment. This addition is motivated by a recent study that suggested a ring vaccination of all susceptible individuals in a prescribed area around an outbreak of an EVD (Refs.14,19). This is used, for example, in the control strategy of Smallpox eradication program which could help also to reduce Ebola transmission. As a matter of fact, in March 2015, the ministry of Health in Guinea started a ring vaccination trial, and interim trial results suggested that the vaccine could have a high level of efficacy in humans (Ref. 19). Other viable Ebola vaccine candidates are mentioned in Ref. 13.

(iv) We take into consideration the awareness of the population and their behavioral change for self-protection by adding a compartment of trained individuals. Some public health educational interventions, including the creation of Ebola response teams in local communities and preparedness at household level to minimize the transmission of EVD, are suggested in Ref. 1.

The model is carefully analysed to get insight on the impact of all these sets of educational interventions. Under these interventions, we observe that the number of infectious individuals is much smaller than that obtained in Ref. 7. It was already observed in Ref. 7 that the use of standard numerical methods could produce unreasonable discrete solutions such as negative ones. On the contrary, the nonstandard finite difference (NSFD) method has the potential to replicate the dynamics of the continuous model (Refs. 4,5,27,28,31). Using the Mickens' rules (Refs. 7,26), we have constructed a NSFD scheme, which is dynamically consistent with EVD model.

The rest of the paper is organized as follows. The model is formulated in Section 2. In Section 3, the quantitative and qualitative analysis of the model is given. The sensitivity analysis of the model is presented in Section 4. In Section 5, we propose a nonstandard finite difference scheme for the continuous model. Numerical simulations and concluding remarks are given in Sections 6 and 7, respectively. 


\section{Model formulation}

In this section, we extend the model for the EVD in Ref. 7 by incorporating vaccination and behavioral change of susceptible individuals. The following assumptions are made. The population under consideration is grouped into disjoint classes or compartments. Individuals who are susceptible to EVD are grouped in the $S$ class. Ebola infected individuals are placed in class $I$. The $R$ class contains individuals recovered from EVD. Individuals who receive public health training on how to prevent themselves from contracting Ebola and vaccinated individuals are categorised as the classes $T$ and $V$, respectively. The class of EVD deceased individuals is represented by $D$, while $P$ denotes the Ebola virus concentration in the environment due to shedding by infectious and Ebola deceased individuals. The flow diagram of the transmission dynamics of the EVD is given in Fig. 1.

The corresponding system of nonlinear differential equations is

$$
\begin{aligned}
\frac{d S}{d t} & =\Pi-(\epsilon e+\lambda+\nu+\mu) S, \\
\frac{d I}{d t} & =\lambda S-(\gamma+\delta+\mu) I+(1-\theta) \lambda T, \\
\frac{d R}{d t} & =\gamma I-\mu R \\
\frac{d T}{d t} & =\epsilon e S-(1-\theta) \lambda T-\mu T \\
\frac{d V}{d t} & =\nu S-\mu V \\
\frac{d D}{d t} & =(\delta+\mu) I-b D \\
\frac{d P}{d t} & =\xi I+\alpha D-\eta P
\end{aligned}
$$

where

$$
N(t)=S(t)+I(t)+R(t)+T(t)+V(t)
$$

is the total population at time $t \geq 0$. All parameters are nonnegative and they are described in Table 1. The force of infection $\lambda$, the behavioral change function $e$ and the governing equation for the evolution of the virus concentration in the environment deserve more specific descriptions and comments as outlined below. Susceptible individuals $S$ may acquire infection after effective contacts with infectious individuals $I$, EVD deceased human bodies $D$ and contaminated environment $P$ at the rates $\beta_{1}, \beta_{2}$ and $\beta_{3}$, respectively. While the first source of infection of susceptible is typically formulated by standard incidence (Ref. 15), a couple of options are available in the literature regarding the other two sources. For instance, in Ref. 36 , indirect transmission through environment occurs in a saturated manner and takes the form

$$
\frac{\beta_{3} S P}{K+P}
$$




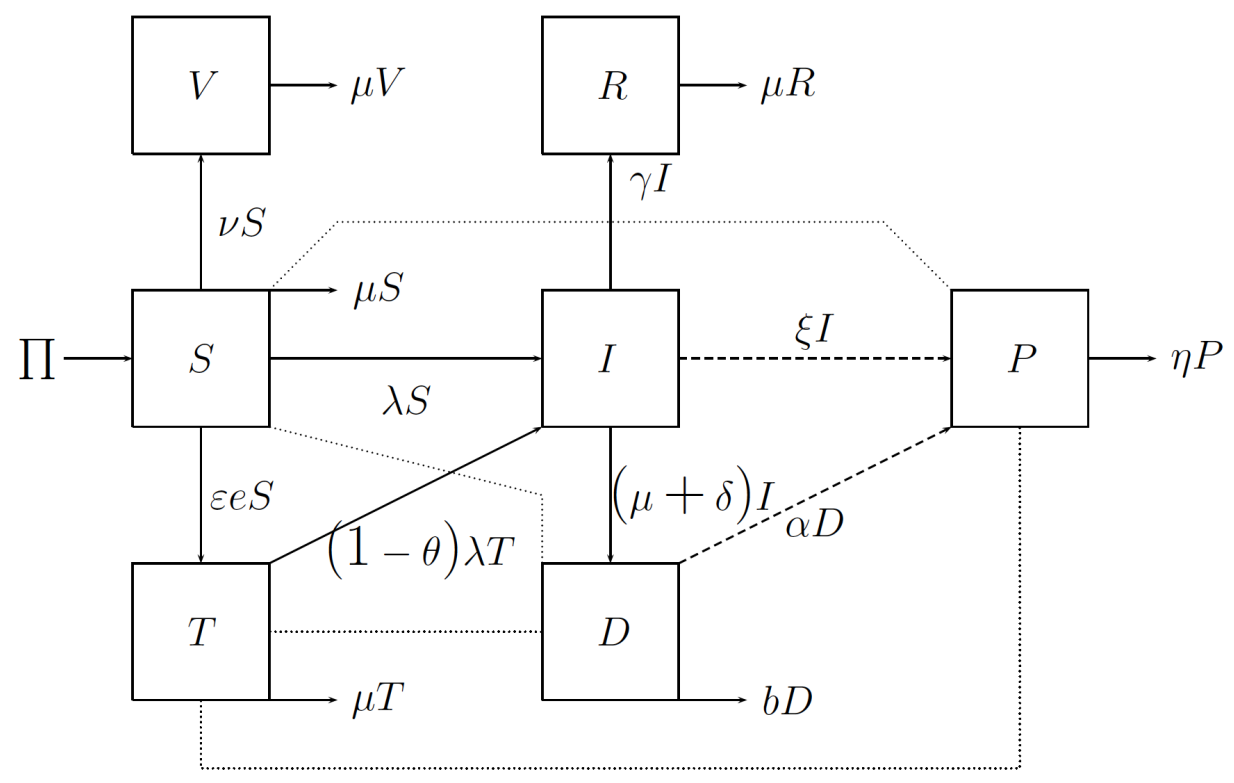

Fig. 1. Schematic diagram of EVD transmission dynamics.

where $K$ is the concentration of the disease pathogen in the environment which increases $50 \%$ chance of triggering the disease transmission.

In the present work, we follow Ref. 32 and formulate the force of infection as

$$
\lambda=\frac{\beta_{1} I}{N}+\beta_{2} D+\beta_{3} P,
$$

with mass action incidence for the last two terms. Thus, (2.3) extends the formulation in Ref. 7 where the mass action principle is used for the three sources of infection.

As noted in Ref. 18, human learning behavior does not keep on increasing for ever. After a sharp increase, the rate of increase in the awareness function $e$ slows down and settles to some value asymptotically. Therefore, we define the awareness function $e(\lambda)$ by the Hill-type function in terms of the force of infection

$$
e(\lambda)=\frac{\lambda^{n}}{\lambda_{0}^{n}+\lambda^{n}},
$$

where $\lambda_{0}$ is the value of the force of infection corresponding to the threshold infectivity in which the individuals start reacting swiftly. At the beginning of an EVD outbreak, individuals understand very little about it; there could be no reaction and this can be related to the situation at the disease-free equilibrium such that 


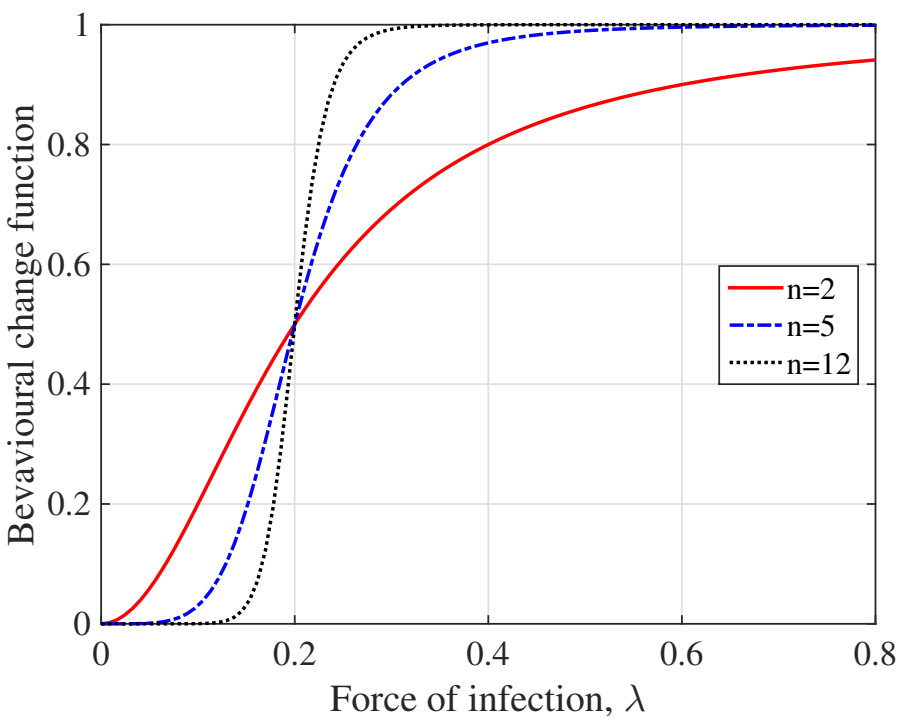

Fig. 2. Change of behavior with respect to the force of infection for $\lambda_{0}=0.2$.

$e(\lambda)=e(0)=0$. However, as the risk of the disease increases, individuals start to think of the type of measures to take in order to avoid all means of contracting the disease. These protection measures, if perfect, account for an increase in the values of $e$ to unity. The order $n$ of the function $e(\lambda)$ is a Hill coefficient that portrays the rate of reaction by the population. See Fig. 2 for the relation between $e$ and the force of infection $\lambda$.

It is possible to add in the last equation of (2.1) a recruitment rate function $\sigma$ of the disease pathogens in the environment. The recruitment $\sigma$ could be a logistic growth function, specifically in the case of free-living pathogens such as bacteria (Refs. 5,6). It could also be a positive constant, implying then that the disease will persist (Ref. 7). In this paper, we assume that $\sigma=0$, resulting in the existence of a disease-free equilibrium, the stability analysis of which will be considered in the next section.

The system (2.1) is appended with the following nonnegative initial conditions: $S(0)=S_{0}, I(0)=I_{0}, R(0)=R_{0}, T(0)=T_{0}, V(0)=V_{0}, D(0)=D_{0}$ and $P(0)=P_{0}$.

\section{Quantitative and qualitative analysis}

We start with the well-posedness result which is stated as follows.

Theorem 3.1 The Ebola model (2.1) is a dynamical system on the biologically 


\begin{tabular}{cl}
\hline Parameter & Description \\
\hline$\Pi$ & Recruitment rate of susceptible individuals \\
$\mu$ & Rate of recovery \\
$\delta$ & Natural death rate \\
$\epsilon$ & Rbola-induced death rate \\
$\nu$ & Rate of vaccination \\
$\frac{1}{b}$ & Mean caring duration of Ebola deceased human individuals \\
$\alpha$ & Shedding rate of Ebola deceased individuals to the environment \\
$\xi$ & Shedding rate of infectious individuals to the environment \\
$\theta$ & Average effectiveness of existing self-preventive measures \\
$\eta$ & Decay rate of Ebola virus in the environment \\
$\beta_{1}$ & Contact rate to the $I$ class \\
$\beta_{2}$ & Contact rate to the $D$ class \\
$\beta_{3}$ & Contact rate to the $P$ class \\
\hline
\end{tabular}

Table 1. Description of parameters of the model (2.1).

feasible region

$$
\begin{aligned}
\Omega= & \left\{(S, I, R, T, V, D, P) \in \mathbb{R}_{+}^{7}: 0 \leq S(t)+I(t)+R(t)+T(t)+V(t)=N(t) \leq \frac{\Pi}{\mu},\right. \\
& \left.D(t) \leq \frac{(\mu+\delta) \Pi}{b \mu} \text { and } P(t) \leq \frac{1}{\eta}\left(\frac{\xi \Pi}{\mu}+\frac{\alpha(\mu+\delta) \Pi}{b \mu}\right)\right\} .
\end{aligned}
$$

The proof of Theorem 3.1, based on the method mentioned in Ref. 9 to show positivity and Theorem 2.1.5 in Ref. 34, is given in Appendix A.

Next, we determine the equilibrium solutions of the system (2.1). We set the right-hand side of (2.1) equal to zero:

$$
\begin{aligned}
\Pi-(\epsilon e+\lambda+\nu+\mu) S & =0, \\
\lambda S-(\gamma+\delta+\mu) I+(1-\theta) \lambda T & =0, \\
\gamma I-\mu R & =0, \\
\epsilon e S-(1-\theta) \lambda T-\mu T & =0, \\
\nu S-\mu V & =0, \\
(\mu+\delta) I-b D & =0, \\
\xi I+\alpha D-\eta P & =0 .
\end{aligned}
$$

Then, the disease-free equilibrium (DFE) is

$$
E_{0}=(S, I, R, T, V, D, P)=\left(\frac{\Pi}{\mu+\nu}, 0,0,0, \frac{\nu \Pi}{\mu(\mu+\nu)}, 0,0\right) .
$$

At the disease-free equilibrium, the behavioral change function $e(\lambda)=e(0)=0$. 
The basic reproduction number, which is very important for the qualitative analysis of the model, is determined by using the method of the next generation matrix in Refs. 12,38.

For the model under consideration, using the notation $X=(I, D, P)$, we have the vector functions

$$
\mathcal{F}(X)=\left(\begin{array}{c}
\lambda S+(1-\theta) \lambda T \\
0 \\
0
\end{array}\right) \quad \text { and } \quad \mathcal{U}(X)=\left(\begin{array}{c}
(\mu+\delta+\gamma) I \\
-(\mu+\delta) I+b D \\
-\xi I-\alpha D+\eta P
\end{array}\right)
$$

representing the rates at which disease compartments increase and decrease in size due to the disease, respectively. The next generation matrix is given by

$$
\mathcal{B}=J_{\mathcal{F}} J_{\mathcal{U}}^{-1}
$$

where

$$
J_{\mathcal{F}}=\left(\begin{array}{ccc}
\frac{\mu \beta_{1}}{\mu+\nu} & \frac{\beta_{2} \Pi}{\mu+\nu} & \frac{\beta_{3} \Pi}{(\mu+\nu)} \\
0 & 0 & 0 \\
0 & 0 & 0
\end{array}\right) \quad \text { and } \quad J_{\mathcal{U}}=\left(\begin{array}{ccc}
\mu+\delta+\gamma & 0 & 0 \\
-(\mu+\delta) & b & 0 \\
-\xi & -\alpha & \eta
\end{array}\right)
$$

are the Jacobian matrices of $\mathcal{F}$ and $\mathcal{U}$ at $E_{0}$, respectively.

The basic reproduction number, denoted by $\mathcal{R}_{0}$, is defined as the average number of secondary cases produced in a completely susceptible population by a typical Ebola virus infected individual (i.e., alive or deceased but not buried) during its entire period of being infectious (Ref. 38). Mathematically, $\mathcal{R}_{0}$ is the spectral radius of $\mathcal{B}$ in (3.3), which is explicitly found to be

$$
\mathcal{R}_{0}=\frac{\mu \beta_{1}}{(\nu+\mu)(\gamma+\delta+\mu)}+\frac{(\delta+\mu) \beta_{2} \Pi}{(\nu+\mu) b(\gamma+\delta+\mu)}+\frac{(b \xi+\alpha(\delta+\mu)) \beta_{3} \Pi}{b \eta(\nu+\mu)(\gamma+\delta+\mu)} .
$$

Remark 3.1 A few comments are in order with regard to (3.5). The basic reproduction number in (3.5) consists of the three contributions of the infectious class I, the deceased class $D$ and the Ebola virus concentration in the environment compartment $P$, respectively. It is clear that increasing the vaccination rate $\nu$, increasing the decay rate $\eta$ of Ebola in the environment and decreasing the shedding rates $\xi$ and $\alpha$ from the infectious and deceased compartments will decrease the value of $\mathcal{R}_{0}$.

In Ref. 7, where the mass action incidence formulation, i.e.,

$$
\lambda=\beta_{1} I+\beta_{2} D+\beta_{3} P
$$

is used, the basic reproduction number is

$$
\mathcal{R}_{0}^{M A}=\frac{\beta_{1} \Pi}{\mu(\gamma+\delta+\mu)}+\frac{(\delta+\mu) \beta_{2} \Pi}{b \mu(\gamma+\delta+\mu)}+\frac{(b \xi+\alpha(\delta+\mu)) \beta_{3} \Pi}{b \mu \eta(\gamma+\delta+\mu)} .
$$

By adding self-protection interventions (e.g vaccination) to the setting of Ref. 7 , the threshold quantity $\mathcal{R}_{0}^{M A}$ is improved to

$$
\mathcal{R}_{0}^{M A V}=\frac{\beta_{1} \Pi}{(\nu+\mu)(\gamma+\delta+\mu)}+\frac{(\delta+\mu) \beta_{2} \Pi}{(\nu+\mu) b(\gamma+\delta+\mu)}+\frac{(b \xi+\alpha(\delta+\mu)) \beta_{3} \Pi}{(\nu+\mu) b \eta(\gamma+\delta+\mu)}
$$


8 Berge, Chapwanya, Lubuma and Terefe

in the sense that $\mathcal{R}_{0}^{M A V} \leq \mathcal{R}_{0}^{M A}$. Furthermore, the advantage of self-protection interventions is captured by the fact that the contribution of the deceased class $D$ and the Ebola virus class $P$ to $\mathcal{R}_{0}$ in (3.5) is much less than their counterparts in $\mathcal{R}_{0}^{M A}$.

Theorem 3.2 The disease-free equilibrium point $E_{0}$ given in (3.2) is locally asymptotically stable $(L A S)$ if $\mathcal{R}_{0}<1$ and unstable when $\mathcal{R}_{0}>1$.

The proof of Theorem 3.2 is given in Appendix A. Furthermore, based on KamgangSallet Stability Theorem (Ref. 17), we have proved the next result which improves Theorem 3.2.

Theorem 3.3 The disease-free equilibrium $E_{0}$ is globally asymptotically stable (GAS) if $\mathcal{R}_{0} \leq 1$.

To determine the endemic equilibria of (2.1), let

$$
E^{*}=\left(S^{*}, I^{*}, R^{*}, T^{*}, V^{*}, D^{*}, P^{*}\right)
$$

denote an equilibrium point. Then from (3.1), we obtain the relations

$$
\begin{aligned}
S^{*} & =\frac{\Pi}{\epsilon e^{*}+\lambda^{*}+\nu+\mu}, \quad T^{*}=\frac{\epsilon e^{*}}{(1-\theta) \lambda^{*}+\mu} S^{*}, \quad V^{*}=\frac{\nu}{\mu} S^{*}, \\
I^{*} & =\frac{\lambda^{*}}{\gamma+\delta+\mu}\left(1+\frac{(1-\theta) \epsilon e^{*}}{(1-\theta) \lambda^{*}+\mu}\right) \frac{\Pi}{\epsilon e^{*}+\lambda^{*}+\nu+\mu}, \\
R^{*} & =\frac{\gamma I^{*}}{\mu}, \quad D^{*}=\frac{\mu+\delta}{b} I^{*}, \quad P^{*}=\frac{1}{b \eta}[b \xi+\alpha(\mu+\delta)] I^{*}, \\
N^{*} & =\frac{1}{\mu}\left[\Pi-\delta I^{*}\right],
\end{aligned}
$$

where

$$
e^{*}=\frac{\left(\lambda^{*}\right)^{n}}{\lambda_{0}^{n}+\left(\lambda^{*}\right)^{n}} \text { and } \lambda^{*}=\frac{\beta_{1} I^{*}}{N^{*}}+\beta_{2} D^{*}+\beta_{3} P^{*}
$$

By incorporating (3.9) into (3.10) and doing some algebraic manipulations, we derive the $(2 n+5)$ degree polynomial in $\lambda^{*}$

$$
Q\left(\lambda^{*}\right)=\Pi \lambda^{*} H\left(\lambda^{*}\right),
$$

where

$$
\begin{aligned}
H\left(\lambda^{*}\right) & =\left(\lambda^{*}\right)^{2 n}\left[A_{4}\left(\lambda^{*}\right)^{4}+A_{3}\left(\lambda^{*}\right)^{3}+A_{2}\left(\lambda^{*}\right)^{2}+A_{1} \lambda^{*}+A_{0}\right] \\
& +\left(\lambda^{*}\right)^{n}\left[B_{4}\left(\lambda^{*}\right)^{4}+B_{3}\left(\lambda^{*}\right)^{3}+B_{2}\left(\lambda^{*}\right)^{2}+B_{1} \lambda^{*}+B_{0}\right] \\
& +(\lambda)_{0}^{2 n}\left[C_{4}\left(\lambda^{*}\right)^{4}+C_{3}\left(\lambda^{*}\right)^{3}+C_{2}\left(\lambda^{*}\right)^{2}+C_{1} \lambda^{*}+C_{0}\right]
\end{aligned}
$$


with coefficients

$$
\begin{aligned}
& A_{4}=k_{5}(1-\theta)^{2}(\mu+\gamma)>0, \\
& A_{3}=k_{5}(1-\theta)\left[\left((1-\theta)\left(2 \epsilon+k_{6}\right)+2 \mu\right)(\mu+\gamma)+k_{5} k_{6}(1-\theta)\left(1-\mathcal{R}_{0}\right)\right] \text {, } \\
& +\delta k_{3} \Pi(1-\theta)^{2}, \\
& A_{2}=2 \delta k_{3}(1-\theta)[\mu+(1-\theta) \epsilon] \Pi+k_{5}^{2}\left[(1-\theta)\left(\epsilon+k_{6}\right)\left((1-\theta)\left(\epsilon+k_{6}\right)+4 \mu\right)+\mu^{2}\right] \\
& -k_{5}\left[(1-\theta)\left(\delta(\mu+(1-\theta) \epsilon)\left(\epsilon+k_{6}\right)+\mu k_{4}\right)\right] \\
& -k_{5}\left[\left((1-\theta) k_{4}+\mu \delta\right)\left((1-\theta)\left(2 \epsilon+k_{6}\right)+\mu\right)\right] \text {, } \\
& A_{1}=\delta k_{3} \Pi((1-\theta) \epsilon+\mu)^{2}+k_{5}^{2}\left[2 \mu\left(\epsilon+k_{6}\right)\left((1-\theta)\left(\epsilon+k_{6}\right)+\mu\right)\right] \\
& -k_{5}\left[\left(\epsilon+k_{6}\right)\left((1-\theta) k_{4}+\mu \delta\right)(\mu+(1-\theta) \epsilon)+\mu k_{4}\left((1-\theta)\left(2 \epsilon+k_{6}\right)+\mu\right)\right], \\
& A_{0}=k_{5}^{2} \mu\left(\epsilon+k_{6}\right)\left[\mu(\nu+\mu)\left(1-\mathcal{R}_{0}\right)+\epsilon \mu-\epsilon(1-\theta)(\nu+\mu) \mathcal{R}_{0}\right] \text {, } \\
& B_{4}=2 k_{5} \lambda_{0}^{n}(1-\theta)^{2}(\mu+\gamma)>0 \text {, } \\
& B_{3}=2 \lambda_{0}^{n} \delta k_{3} \Pi(1-\theta)^{2}+2 \lambda_{0}^{n} k_{5}(1-\theta)\left[(1-\theta)\left(\epsilon+2 k_{6}\right)+2 \mu\right] \\
& -2 \lambda_{0}^{n} k_{5}(1-\theta)\left[\delta\left((1-\theta)\left(\epsilon+k_{6}\right)+2 \mu\right)+(1-\theta) k_{4}\right] \text {, } \\
& B_{2}=2 \delta k_{3}(1-\theta) \lambda_{0}^{n} \Pi[2 \mu+(1-\theta) \epsilon]+2 \lambda_{0}^{n} k_{5}^{2}(1-\theta)\left[k_{6}(1-\theta)\left(\epsilon+k_{6}\right)+2 \mu\left(\epsilon+2 k_{6}\right)\right] \\
& -k_{5} \lambda_{0}^{n}\left[(1-\theta)\left[\delta\left[\mu\left(\epsilon+k_{6}\right)+(\mu+(1-\theta) \epsilon) k_{6}\right]+2 \mu k_{4}\right]\right] \\
& -k_{5} \lambda_{0}^{n}\left[2\left((1-\theta) k_{4}+\mu \delta\right)\left((1-\theta)\left(\epsilon+k_{6}\right)+\mu\right)\right] \text {, } \\
& B_{1}=2 \mu \delta k_{3} \lambda_{0}^{n} \Pi(\mu+(1-\theta) \epsilon)+2 \mu k_{5}^{2} \lambda_{0}^{n}\left[2(1-\theta)\left(\epsilon+k_{6}\right) k_{6}+\mu\left(\epsilon+2 k_{6}\right)\right] \\
& -k_{5} \lambda_{0}^{n}\left[\left((1-\theta) k_{4}+\mu \delta\right)\left(\mu\left(\epsilon+k_{6}\right)+(\mu+(1-\theta) \epsilon) k_{6}\right)+\right] \\
& -2 k_{5} \lambda_{0}^{n} \mu k_{4}\left((1-\theta)\left(\epsilon+k_{6}\right)+\mu\right) \text {, } \\
& B_{0}=2 \mu^{2} k_{5}^{2} k_{6} \lambda_{0}^{n}\left(\epsilon+k_{6}\right)-\mu k_{4} k_{5} \lambda_{0}^{n}\left[\mu\left(\epsilon+k_{6}\right)+(\mu+(1-\theta) \epsilon) k_{6}\right], \\
& C_{4}=k_{5}(1-\theta)^{2}(\mu+\gamma)>0 \text {, } \\
& C_{3}=\delta k_{3} \Pi(1-\theta)^{2}+2 k_{5}^{2}(1-\theta)\left[(1-\theta) k_{6}+\mu\right] \\
& -k_{5}(1-\theta)\left[\delta\left[(1-\theta) k_{6}+\mu\right]+\left[(1-\theta) k_{4}+\mu \delta\right]\right], \\
& C_{2}=2 \mu \delta k_{3} \Pi(1-\theta)+k_{5}^{2}\left[(1-\theta)^{2} k_{6}+\mu^{2}+4 \mu(1-\theta) k_{6}\right] \\
& -k_{5}\left[(1-\theta) \mu\left(k_{4}+\delta k_{6}\right)+\left[(1-\theta) k_{4}+\mu \delta\right]\left[(1-\theta) k_{4}+\mu\right]\right], \\
& C_{1}=\delta \mu^{2} k_{3} \Pi+2 \mu k_{5}^{2} k_{6}\left[(1-\theta) k_{6}+\mu\right]-\mu k_{5}\left[k_{6}\left[(1-\theta) k_{4}+\mu \delta\right]+k_{4}\left[(1-\theta) k_{6}+\mu\right]\right] \text {, } \\
& C_{0}=\mu^{2} k_{5}^{2} k_{6}^{2}\left(1-\mathcal{R}_{0}\right) \text {, }
\end{aligned}
$$

and

$$
k_{4}=k_{5} k_{6} \mathcal{R}_{0}, \quad k_{5}=\gamma+\delta+\mu \quad \text { and } \quad k_{6}=\nu+\mu .
$$

Thus, $\lambda^{*}$ is a non-negative real root of $Q\left(\lambda^{*}\right)$ and $\lambda^{*}=0$ gives the disease-free equilibrium. When $\mathcal{R}_{0}>1$, we have $C_{0}<0$. Since $H\left(\lambda^{*}\right)$ is a polynomial of even degree $(2 n+4)$ with leading $\left(A_{4}>0\right)$ and constant $\left(C_{0}<0\right)$ terms of opposite signs, there is always an odd number of sign changes on its non-zero coefficients. In line with Descartes' rule of signs, the existence of at least one positive root is guaranteed. Thus, the existence part of the following theorem, its stability part being proved by the Center Manifold Theory (Ref. 10) in Appendix A. 
Theorem 3.4 When $\mathcal{R}_{0}>1$, the Ebola model (2.1) has at least one endemic equilibrium point, which is locally asymptotically stable.

Theorem 3.3 already implies that there is no LAS endemic equilibria when $\mathcal{R}_{0}<$ 1 and we will use the Center Manifold Theory (Ref. 10) in Appendix A to confirm the following result.

Theorem 3.5 The model (2.1) does not undergo a backward bifurcation at $\mathcal{R}_{0}=1$.

Let us now address the severity of the disease when $\mathcal{R}_{0}>1$.

Theorem 3.6 Let $I^{*}(\nu, \epsilon)$ denote the infectious component of the endemic equilibrium point corresponding to the parameters $\nu$ and $\epsilon$. Then $I^{*}(\nu, \epsilon)<I^{*}(0,0)$.

Proof. At the endemic equilibrium point $E^{*}=\left(S^{*}, I^{*}, R^{*}, T^{*}, V^{*}, D^{*}, P^{*}\right)$, the conservation law in Eq. (A.1) becomes

$$
\Pi-\mu N^{*}-\delta I^{*}=0 \text { where } N^{*}=S^{*}+I^{*}+R^{*}+T^{*}+V^{*} .
$$

Hence,

$$
I^{*}(\nu, \epsilon)=\frac{\Pi-\mu\left(S^{*}+R^{*}+T^{*}+V^{*}\right)}{\delta+\mu}<\frac{\Pi-\mu\left(S^{*}+R^{*}\right)}{\delta+\mu}=I^{*}(0,0) .
$$

This completes the proof.

\section{Sensitivity analysis}

The basic reproduction number $\mathcal{R}_{0}$ is an important quantity that depends on the parameters involved in the system of differential equations (2.1). In this section, we would like to know how $\mathcal{R}_{0}$ responds to the changes in the parameters. The change in the value of $\mathcal{R}_{0}$ with respect to changes in the values of the parameters is measured by the derivative of this quantity with respect to that parameter. Mathematically, the sensitivity of $\mathcal{R}_{0}$ with respect to a parameter $p$ is given by Ref. 25 ,

$$
\gamma_{p}^{\mathcal{R}_{0}}=\frac{\partial \mathcal{R}_{0}}{\partial p}
$$

A much more powerful tool is the normalized sensitivity index of $\mathcal{R}_{0}$, which measures the change in the value of $\mathcal{R}_{0}$ with respect to the change in the parameter $p$. It is given by

$$
\mathcal{E}_{p}^{\mathcal{R}_{0}}=\frac{\partial \mathcal{R}_{0}}{\partial p} \frac{p}{\mathcal{R}_{0}}=\frac{\triangle \mathcal{R}_{0} \%}{\triangle p \%} .
$$

Hence, if $p$ changes by $y \%$, then $\mathcal{R}_{0}$ will change by $\mathcal{E}_{p}^{\mathcal{R}_{0}} y \%$. The sensitivity index of $\mathcal{R}_{0}$ with respect to the parameter $p$ is positive if $\mathcal{R}_{0}$ is increasing with respect to $p$ and negative if $\mathcal{R}_{0}$ is decreasing with respect to $p$. The calculation of sensitivity indices of $\mathcal{R}_{0}$ at the baseline parameter values of the model is given in Table 2.

By using the reproduction number $\mathcal{R}_{0}$ as the response function, Table 2 can be used to propose effective control strategies to avoid direct and indirect contacts 


\begin{tabular}{cccc}
\hline Parameter & Baseline Value & Sensitivity index & Source \\
\hline$\beta_{1}$ & 0.6 & 0.0013 & Ref. 11 \\
$\beta_{2}$ & 0.12 & 0.0099 & Ref. 37 \\
$\beta_{3}$ & 0.01 & 0.9888 & Assumed \\
$\alpha$ & 0.04 & 0.5493 & Assumed \\
$\xi$ & 0.04 & 0.4395 & Assumed \\
$\Pi$ & 30 & 0.9987 & Assumed \\
$b$ & 0.4 & -0.5592 & Ref. 21 \\
$\eta$ & 0.003 & -0.9888 & Ref. 8 \\
$\delta$ & 0.48 & -0.3203 & Ref. 2 \\
$\nu$ & 0.005 & -0.2000 & Assumed \\
$\mu$ & 0.02 & -0.8021 & Ref. 29 \\
$\gamma$ & 0.06 & -0.1071 & Ref. 11 \\
\hline
\end{tabular}

Table 2. Table for the sensitivity index of $\mathcal{R}_{0}$ with respect to each parameter in (3.5).

with the potential Ebola virus sources. It is evident, from Table 2, that $\eta$ is the most sensitive parameter in the control of EVD. This will be discussed further in the numerical simulations.

\section{NSFD scheme}

Given the rich dynamics of the model (2.1) and the well-documented shortcomings of standard numerical methods to correctly replicate these dynamics (Refs. 4,5,27,28, 31), we design in this section a NSFD scheme. This is done by using Mickens' rules on complex denominator function of discrete derivative and nonlocal approximation of nonlinear terms (Refs. 4,26).

Let $Y_{k}=\left(S_{k}, I_{k}, R_{k}, T_{k}, V_{k}, D_{k}, P_{k}\right)$ denote an approximation of $Y\left(t_{k}\right)$ at the discrete time $t_{k}=k \Delta t$ where $k \in \mathbb{N}$ and $h=\Delta t>0$ is the step size. We propose the NSFD scheme

$$
\begin{aligned}
\frac{S_{k+1}-S_{k}}{\phi} & =\Pi-\left(\epsilon e_{k}+\lambda_{k}+\nu+\mu\right) S_{k+1}, \\
\frac{I_{k+1}-I_{k}}{\phi} & =\lambda_{k} S_{k+1}-(\gamma+\delta+\mu) I_{k+1}+(1-\theta) \lambda_{k} T_{k+1}, \\
\frac{R_{k+1}-R_{k}}{\phi} & =\gamma I_{k+1}-\mu R_{k+1}, \\
\frac{T_{k+1}-T_{k}}{\phi} & =\epsilon e_{k} S_{k+1}-\left((1-\theta) \lambda_{k}+\mu\right) T_{k+1}, \\
\frac{V_{k+1}-V_{k}}{\phi} & =\nu S_{k+1}-\mu V_{k+1}, \\
\frac{D_{k+1}-D_{k}}{\phi} & =(\mu+\delta) I_{k+1}-b D_{k+1}, \\
\frac{P_{k+1}-P_{k}}{\phi} & =\xi I_{k}+\alpha D_{k}-\eta P_{k+1},
\end{aligned}
$$


where the complex denominator function

$$
\phi=\phi(h)=\frac{1-e^{-(\gamma+\delta+\mu) h}}{\gamma+\delta+\mu}
$$

satisfies the asymptotic property

$$
\phi(h)=h+\mathcal{O}\left(h^{2}\right) .
$$

The nonlocal approximation of nonlinear terms is reinforced by observing that

$$
\lambda_{k}=\frac{\beta_{1} I_{k}}{N_{k}}+\beta_{2} D_{k}+\beta_{3} P_{k} \quad \text { and } \quad e_{k}=e\left(\lambda_{k}\right)=\frac{\lambda_{k}^{n}}{\lambda_{0}^{n}+\lambda_{k}^{n}} .
$$

The NSFD scheme (5.1) is an extension of the one in Ref. 7, through the form of $\lambda_{k}$ and the presence of $e_{k}, V_{k}$ and $T_{k}$.

By rearranging (5.1), we obtain

$$
\begin{aligned}
S_{k+1} & =\frac{\Pi \phi+S_{k}}{1+\left(\epsilon e_{k}+\lambda_{k}+\nu+\mu\right) \phi}, \\
I_{k+1} & =\frac{\lambda_{k} \phi\left(S_{k+1}+(1-\theta) T_{k+1}\right)+I_{k}}{1+(\gamma+\delta+\mu) \phi}, \\
R_{k+1} & =\frac{\gamma \phi I_{k+1}+R_{k}}{1+\mu \phi}, \\
T_{k+1} & =\frac{\phi \epsilon e_{k} S_{k+1}+T_{k}}{1+\left((1-\theta) \lambda_{k}+\mu\right) \phi}, \\
V_{k+1} & =\frac{\nu \phi S_{k+1}+V_{k}}{1+\mu \phi} \\
D_{k+1} & =\frac{(\delta+\mu) \phi I_{k+1}+D_{k}}{1+b \phi}, \\
P_{k+1} & =\frac{\left(\xi I_{k}+\alpha D_{k}\right) \phi+P_{k}}{1+\eta \phi} .
\end{aligned}
$$

The equivalent form (5.5) has a Gauss-Seidel structure for the computation of the terms one after the other.

By following the ideas in Refs. 4,26, as it is done in Ref. 7, the following results can be proved:

Theorem 5.1 Irrespective of the value of the step size $\triangle t$, the NSFD scheme (5.1) replicates the dynamics of the model (2.1) as stated in Theorems 3.1-3.6.

\section{Numerical simulations}

In this section, numerical simulations for the proposed NSFD scheme (5.1) or (5.5) are given. Parameter values to be used are in Table 2 while $\theta=0.5, \epsilon=0.9$ and , $n=2$.

The global and local asymptotic stability of the disease-free and endemic equilibrium points guaranteed by Theorems 3.3-3.4 are illustrated in Fig. 3 for $\mathcal{R}_{0}=0.68$ and $\mathcal{R}_{0}=8.7$, respectively. The numerical solutions of the proposed model under different protection interventions are presented to highlight their contribution on the reduction of the severity and the endemicity of the disease.

For instance, in Fig. 4 (a), it is observed how interventions reduce the number of infectious individuals on the long run. In accordance with Theorem 3.6, the implementation 
of all interventions stabilises the trajectories at the endemic equilibrium $I^{*}=14$ whereas in the absence of interventions $I^{*}=17$. This represents a $17.6 \%$ reduction in the number of infectious individuals. Additional information about the contribution of education and vaccination interventions on the reduction of infectious individuals is presented in Fig. 4 (b). These findings are made more precise in Fig. 5. Fig. 5(a) illustrates that the endemic equilibrium $I^{*}$ decreases as the vaccination rate $(\nu)$ and decay rate of Ebola virus in the environment $(\eta)$ increase while Fig. $5(\mathrm{~b})$ shows that the endemic equilibrium $I^{*}$ increases as the contact rates $\left(\beta_{1}, \beta_{2}\right.$ and $\left.\beta_{3}\right)$ increase. In particular, the contact rate with the environment $\left(\beta_{3}\right)$, contributes to a significant increase in $I^{*}$ compared to the other contact rates. By considering the mass action principle with self protection interventions, the endemic equilibrium $I^{*}$ is less than that of the mass action principle without interventions, as illustrated in Fig. 6(a). This is in line with Remark 3.1. Finally, in Fig. 6(b), we observe the inverse relationship between the number of infective individuals and the self-preventive measure parameter $\theta$.

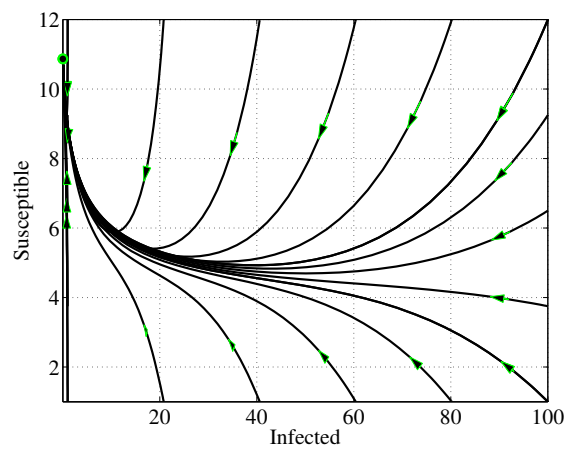

(a)

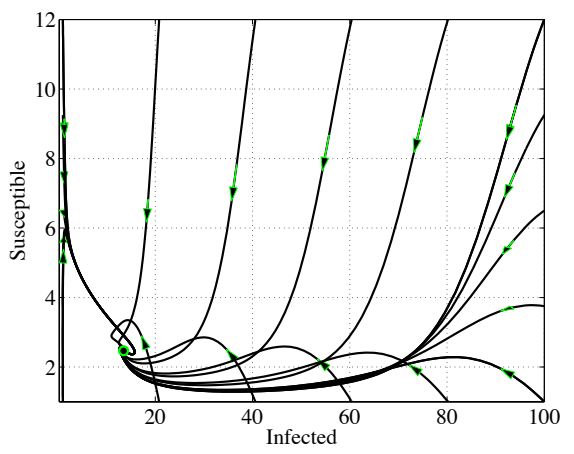

(b)

Fig. 3. a) GAS of the disease-free equilibrium. b) LAS of the endemic equilibrium point.

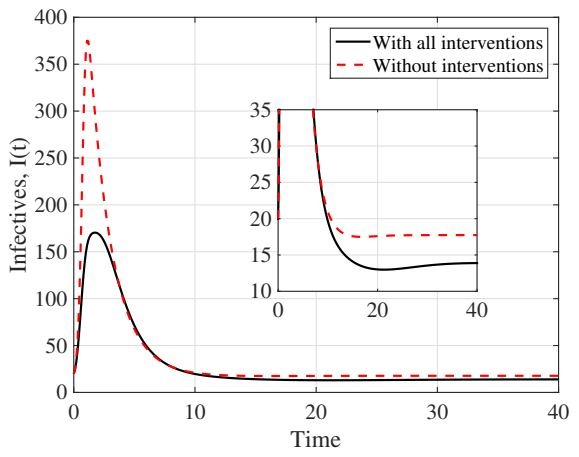

(a)

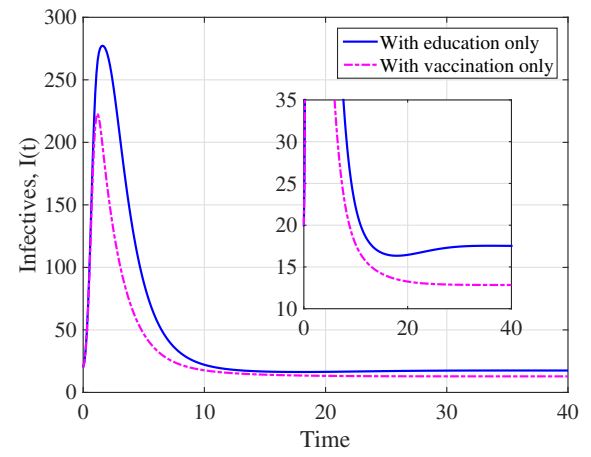

(b)

Fig. 4. (a) Number of infectious with and without interventions (b) Number of infectious with education and vaccination interventions. 


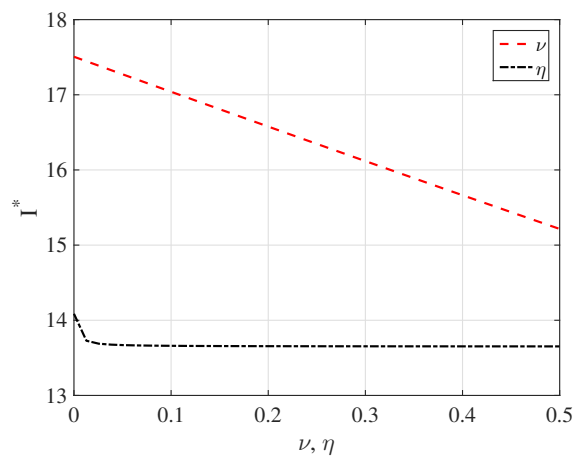

(a)

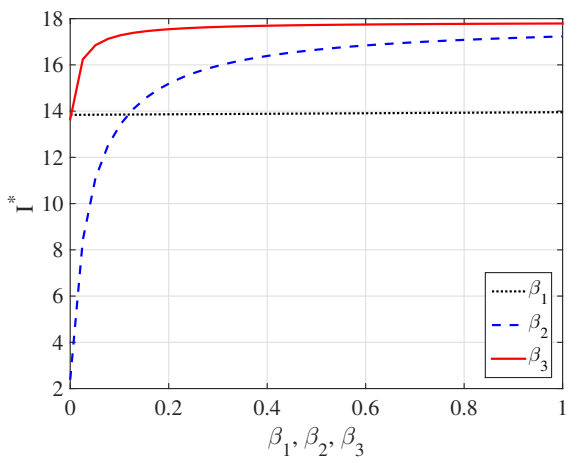

(b)

Fig. 5. (a): Sensitivity analysis with respect to $\nu$ and $\eta$. (b): Sensitivity analysis with respect to $\beta_{1}, \beta_{2}$ and $\beta_{3}$.

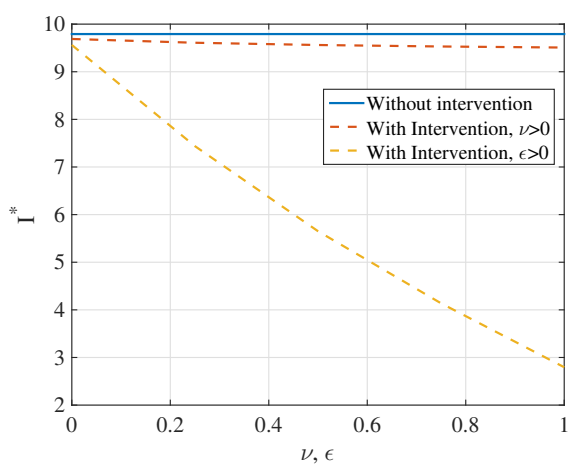

(a)

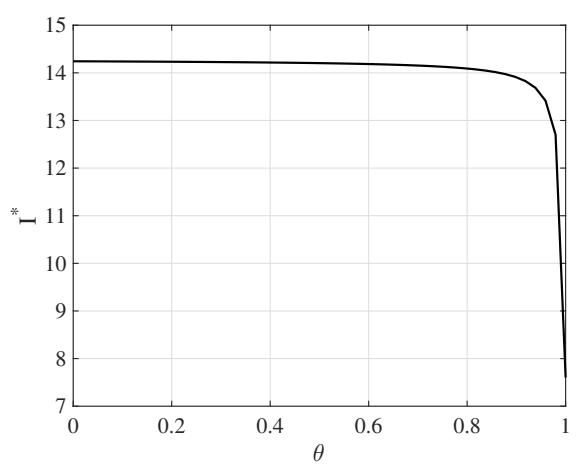

(b)

Fig. 6. (a) Mass action with and without self protection interventions. (b) The relation between $I^{*}$ and self-preventive measure $\theta$.

\section{Conclusion}

The transmission dynamics of the Ebola outbreak in the Central and Western Africa has been studied in Refs. 1, 2, 16, 21, 29 by using the mass action formulation but without the environment class and the vital dynamics of the population. A mathematical model proposed in Ref. 7 incorporated the vital dynamics and the contribution of the environment in the transmission dynamics of the EVD. Here, we have enriched the work in Ref. 7 by widening the educational interventions on the population and by combining the standard incidence and the mass action principle in the formulation of the force of infection. This resulted in the formulation of model (2.1). In the analysis of the model, we proved the global asymptotic stability of the disease-free equilibrium point when $\mathcal{R}_{0} \leq 1$. The existence and the local asymptotic stability of the endemic equilibrium point are guaranteed for $\mathcal{R}_{0}>$ 
1. The contribution of vaccination and training of susceptible individuals in the control strategy of the EVD are shown analytically and illustrated graphically. More precisely, applying both type of interventions is very important to have less number of infectious individuals which minimize the severity of EVD in the population. Our findings in this study strengthen the recommendations given in Ref. 7 to eradicate the EVD.

The sensitivity indexes of $\mathcal{R}_{0}$ with respect to the parameters involved in the model are computed and the more influential parameters on $\mathcal{R}_{0}$ are identified. For instance, when the contact rate $\beta_{3}$ increases, the basic reproduction number $\mathcal{R}_{0}$ will increase correspondingly. On the other hand, increasing $b, \eta$ or $\nu$ decreases the value of $\mathcal{R}_{0}$. Hence, this study recommends to the health policy makers to take into account the following points in their policies in order to have less damage of Ebola virus outbreak in the population:

(i) avoiding contacts with Ebola virus infectious individuals, Ebola deceased bodies and Ebola contaminated environment,

(ii) minimizing the mean caring duration of deceased human bodies due to EVD,

(iii) increasing the decay rate of Ebola virus in the environment and vaccination rate of individuals who are living in Ebola outbreak areas.

These recommendations can be achieved by using successive training in the population.

From the numerical simulations point of view, our NSFD scheme helps to observe the properties of the continuous model.

Although the above recommendations in (i)-(iii) (if strictly implemented) can be effective to eradicate the disease, it has been observed for the fast outbreaks that additional control measures are needed. As a consequence, possible extension of this work includes:

(1) the incorporation of contact tracing as additional control measure (Refs. 24,30),

(2) the multi-species setting to account for the extreme case where a region is attacked by more than one Ebola virus species,

(3) the incorporation of patches to account for the circulation of the disease in many countries

(4) the use of the theory of optimal control to determine when and to what extent we can control the most influential parameters in the transmission dynamics of the EVD,

(5) the investigation of the uniqueness of the endemic equilibrium and its global asymptotic stability.

\section{Acknowledgments}

The authors acknowledge, with thanks, the support of the South African Research Chairs Initiative of the Department of Science and Technology and National Research Foundation: SARChI Chair in Mathematical Models and Methods in Bioengineering and Biosciences. TB and YAT acknowledge the support, in part, of DST-NRF Centre of Excellence in Mathematical and Statistical Sciences (CoE-MaSS). The authors are grateful to the anonymous reviewers, and the Handling Editor, for their suggestions that have greatly improved the paper.

\section{References}

1. Agusto F. B., Teboh-Ewungkem M. I. and Gumel A. B., Mathematical assessment of the effect of traditional beliefs and customs on the transmission dynamics of the 2014 Ebola outbreaks, BMC Medicine, 13 (2015): 96: 1-17. 
2. Althaus C., Estimating the reproduction number of Ebola virus (EBOV) during outbreak in West Africa, PLOS Currents Outbreaks, 2 September 2014.

3. Anderson R. M., May R. M., et al. Population biology of infectious diseases: Part I. Nature, 280 (2) (1979): 361-367.

4. Anguelov R. and Lubuma J. M. - S., Contribution to the Mathematics of the Nonstandard Finite Difference Methods and Applications, Numerical Methods for Partial Differential Equations, 17 (2001): 518-543.

5. Anguelova R., Dumontb Y. , Lubumaa J.M.-S. and Shillorc M., Dynamically consistent nonstandard finite difference schemes for epidemiological models, Journal of computational and Applied Mathematics, 255 (2014): 161-182.

6. Bani-Yaghoub M., Gautam R. Shuai Z., van den Driessche P. and Ivanek R., Reproduction numbers for infections with free-living pathogens growing in the environment, Journal of Biological Dynamics, 6 (2) (2012): 923-940.

7. Berge T., Lubuma J. M. -S., Moremedi G. M., Morris N. and Shava R. K., A simple mathematical model for Ebola in Africa, Journal of Biological Dynamics, 11 (1) (2016): 42-74.

8. Bibby K., Casson L.W., Stachler E. and Haas C.N., Ebola virus persistence in the environment: state of the knowledge and research needs, Environmental Science and Technology Letters, 2 (2015): 2-6.

9. Busenberg S. and Cooke K., Vertically Transmitted Disease: Models and Dynamics, Springer-Verlag, 23 (1993).

10. Castilo-Chavez C. and Song B., Dynamical models of Tuberculosis and their application, Mathematical Biosciences and Engineering, 1 (2) (2004): 361-404.

11. Chowell G., Hengartner N.W., Castillo-Chavez C., Fenimore P.W., and Hyman J. M. The basic reproductive number of Ebola and the effects of public health measures: The cases of Congo and Uganda, Journal of Theoretical Biology, 229 (2004): 119-126.

12. Diekmann O. and Heesterbeek J., Mathematical epidemiology of infectious diseases, Wiley, 2000.

13. Galvani A. P., Ndeffo-Mbah M. L., Wenzel N. and Chids J. E., Ebola vaccination: if not now when? Annals of Internal Medicine, 161 (10) (2014): 749 -751.

14. Henao-Resrepo A. M., et al., Efficacy and effectiveness of an rVSV-vectored vaccine expressing Ebola surface glycoprotein: interim results from the Guinea ring vaccination cluster-randomised trial, World Health Organization, 386 (2015): 857-866.

15. Hethcote H. W., The Mathematics of Infectious Diseases, SIAM Review, 42 (2000), 599-653.

16. Ivorra B., Ngom D. and Ramos A. M. Be-CoDiS: A mathematical model to predict the risk of human diseases spread between countries-validation and application to the 2014-2015 Ebola virus disease epidemic, Bulletin of Mathematical Biology, 77 (2015): 1668-1704.

17. Kamgang J. C. and Sallet G., Computation of threshold conditions for epidemiological models and global stability of the disease-free equilibrium (DFE), Mathematical Biosciences, 213 (2008): 1-12.

18. Kassa S. M. and Ouhinou A., Epidemiological models with prevalence dependent endogenous self-protection measure, Mathematical Biosciences, 229 (2011): 41-49.

19. Kucharski A. J., Eggo R. M. Watson C. H. Camacho A. Funk S. and Edmunds W. J., Effectiveness of ring vaccination as control strategy for Ebola virus disease, Emerging Infectious Diseases, 22 (1), (2016): 105-108.

20. Lasalle J. P. The stability of dynamical systems, SIAM, Philadelphia, PA, 1976.

21. Legrand J., Grais R. F., Boelle P. Y., Valleron A. J. and Flahault A., Understanding the dynamics of Ebola epidemic, Epidemiology and Infection, 135 (2007): 610-621. 
22. Leroy E .M., Rouquet P., Formenty P., Souquiére S., Kilbourne A., Froment J. -M., Bermejo M., Smit S., Karesh W., Swanepoel R., Zaki S. R. and Rollin P. E., Multiple Ebola virus transmission events and rapid decline of central African wildlife, Science, 303 (5656) (2004): 387-390.

23. Leroy E. M., Kumulungui B., Pourrut X., Rouquet P., Hassanin A., Yaba P., Délicat A., Paweska J. T., Gonzalez J. -P. and Swanepoel R., Fruit bats as reservoirs of Ebola virus, Nature, 438 (2005): 575-576.

24. Maganga G. D, et al., Ebola Virus Disease in the Democratic Republic of Congo, The New England Journal of Medicine, 27 November 2014: 2084-291.

25. Martcheva M. An Introduction to Mathematical Epidemiology, Springer, 2015.

26. Mickens R. E., Nonstandard Finite Difference Models of Differential Equations, World Scientific, Singapore, 1994.

27. Mickens R. E., Applications of Nonstandard Finite Difference Schemes, World Scientific, Singapore, 2000.

28. Mickens R. E., Advances in the Application of Nonstandard Finite Difference Schemes, World Scientific, Singapore, 2005.

29. Ndanguza D., Tchuenche J. M. and Haario H., Statistical data analysis of the 1995 Ebola outbreak in the Democratic Republic of Congo, Afrika Matematika, 24 (2013): $55-68$.

30. Ngwa G. A., and Teboh-Ewungkem M. I., A Mathematical Model with Quarantine States for the Dynamics of Ebola Virus Disease in Humans Population, Computational and Mathematical Methods in Medicine, (2016): 1- 29.

31. Patidar, K. C., Nonstandard finite difference methods: recent trends and further developments, Journal of Difference Equations and Applications, 22 (6) (2016): 817-849.

32. Shu H. and Wang X. -S, Global dynamics of a coupled epidemic models, Discrete and Continuous Dynamical Systems-Series, B, 22 (4) (2017): 1575-1585.

33. Smith H., Monotone Dynamical Systems: An Introduction to the Theory of Competitive and cooperative System, American Mathematical Society, Providence, 1995.

34. Stuart A. M. and Humphries A. R., Dynamical Systems and Numerical Analysis, Cambridge University Press, Cambridge, 1998.

35. Sullivan N., Yang Z. and Nabel G., Ebola virus pathogenesis: Implications for vaccines and therapies, Journal of Virology, September (2003): 9733-9737.

36. Tian J. P. and Wang J., Global stability for cholera epidemic models, Mathematical Biosciences, 232 (2011): 31-41.

37. Towers S., Patterson-Lomba O. and Castillo-Chavez C., Temporal variations in the effective reproduction number of the 2014 West Africa Ebola outbreak, PLOS Current Outbreaks, 18 September 2014.

38. van der Driessche P. and Watmough J., Further notes on the basic reproduction number, in Brauer F., van der Driessche P. and Wu J. (eds), Mathematical epidemiology, Lecture Notes in Mathematical Biosciences Subseries, 1945, Springer, (2008): 159-178.

\section{Appendix A.}

Proof of Theorem 3.1. We want to show that for nonnegative initial data, the system (2.1) possesses at all time $t \geq 0$ a unique nonnegative solution which lies in region $\Omega$. The proof will follow two steps.

Following the approach in Ref. 9, it can be shown that any solution of (2.1) corresponding to nonnegative initial conditions is nonnegative.

In a second step, we have that any solution satisfies some a prior estimates. By adding 
the first five equations of (2.1), we obtain

$$
\frac{d N}{d t}=\Pi-\mu N-\delta I
$$

Thus

$$
\Pi-(\delta+\mu) N \leq \frac{d N}{d t}=\Pi-\mu N-\delta I \leq \Pi-\mu N,
$$

which by applying Gronwall inequality implies

$$
0 \leq N(t) \leq \frac{\Pi}{\mu} \quad \text { for } \quad 0 \leq N_{0} \leq \frac{\Pi}{\mu} .
$$

Since the last two equations of (2.1) imply that

$$
\frac{d D}{d t}=\leq(\delta+\mu) \frac{\Pi}{\mu}-b D \quad \text { and } \quad \frac{d P}{d t} \leq \frac{\xi \Pi}{\mu}+\frac{\alpha(\delta+\mu) \Pi}{b \mu}-\eta P,
$$

another application of Gronwall inequality leads to

$$
0 \leq D(t) \leq \frac{(\delta+\mu) \Pi}{b \mu} \text { for } 0 \leq D_{0} \leq \frac{(\delta+\mu) \Pi}{b \mu}
$$

and

$$
0 \leq P(t) \leq \frac{1}{\eta}\left(\frac{\xi \Pi}{\mu}+\frac{\alpha(\delta+\mu) \Pi}{b \mu}\right) \quad \text { for } \quad 0 \leq P_{0} \leq \frac{1}{\eta}\left(\frac{\xi \Pi}{\mu}+\frac{\alpha(\delta+\mu) \Pi}{b \mu}\right) .
$$

Combining the above two steps and using the well-known result (see Theorem 2.1.5 in Ref. 34), we conclude that (2.1) defines a dynamical system on $\Omega$.

Proof of Theorem 3.2. To show that $E_{0}$ is locally asymptotically stable, we use the Jacobian matrix of the functions at the right-hand side of (2.1) at $E_{0}$. We exclude the equation related to the variable $R$ since the analysis is not affected by this equation. Thus

$$
J_{6}\left(E_{0}\right)=\left(\begin{array}{cccccc}
-(\nu+\mu) & \frac{\mu \beta_{1}}{\nu+\mu} & 0 & 0 & -\frac{\beta_{2} \Pi}{\nu+\mu} & -\frac{\beta_{3} \Pi}{\nu+\mu} \\
0 & \frac{\mu \beta_{1}}{\nu+\mu}-(\gamma+\delta+\mu) & 0 & 0 & \frac{\beta_{2} \Pi}{\nu+\mu} & \frac{\beta_{3} \Pi}{\nu+\mu} \\
0 & 0 & -\mu & 0 & 0 & 0 \\
\nu & 0 & 0 & -\mu & 0 & 0 \\
0 & \delta+\mu & 0 & 0 & -b & 0 \\
0 & \xi & 0 & 0 & \alpha & -\eta
\end{array}\right) .
$$

Expanding the determinant in the characteristic equation $\left|\lambda I_{6}-J_{6}\right|=0$ by the third and fourth columns, where $I_{6}$ is $6 \times 6$ identity matrix, we obtain two eigenvalues $\lambda_{1}=-\mu$ and $\lambda_{2}=-\mu$. The remaining four eigenvalues are those of the matrix

$$
J_{4}=\left(\begin{array}{cccc}
-(\nu+\mu) & \frac{\mu \beta_{1}}{\nu+\mu} & -\frac{\beta_{2} \Pi}{\nu+\mu} & -\frac{\beta_{3} \Pi}{\nu+\mu} \\
0 & \frac{\mu \beta_{1}}{\nu+\mu}-(\gamma+\delta+\mu) & \frac{\beta_{2} \Pi}{\nu+\mu} & \frac{\beta_{3} \Pi}{\nu+\mu} \\
0 & \delta+\mu & -b & 0 \\
0 & \xi & \alpha & -\eta
\end{array}\right) .
$$

The characteristic equation takes the form $\left|\lambda I_{4}-J_{4}\right|=0$ and the expansion along the first column gives a third eigenvalue $\lambda_{3}=-(\nu+\mu)$. Then the remaining eigenvalues are those of the matrix 


$$
J_{3}=\left(\begin{array}{ccc}
\frac{\mu \beta_{1}}{\nu+\mu}-(\gamma+\delta+\mu) & \frac{\beta_{2} \Pi}{\nu+\mu} & \frac{\beta_{3} \Pi}{\nu+\mu} \\
\delta+\mu & -b & 0 \\
\xi & \alpha & -\eta
\end{array}\right) .
$$

The corresponding characteristic equation $\left|\lambda I_{3}-J_{3}\right|=0$ gives the following polynomial in $\lambda$ :

$$
Q(\lambda):=\lambda^{3}+a_{1} \lambda^{2}+a_{2} \lambda+a_{3}=0,
$$

where

$$
\begin{aligned}
a_{1}= & \eta+b+(\gamma+\delta+\mu)\left[1-\mathcal{R}_{0}\right]+\frac{\beta_{2}(\delta+\mu) \Pi}{(\nu+\mu)}+\frac{\beta_{3} \Pi[b \xi+\alpha(\delta+\mu)]}{b \eta(\nu+\mu)}, \\
a_{2}= & \eta b+(\eta+b)(\gamma+\delta+\mu) \times \\
& {\left[1-\mathcal{R}_{0}+\frac{\eta \beta_{2}(\delta+\mu) \Pi}{b(\eta+b)(\nu+\mu)(\gamma+\delta+\mu)}+\frac{\beta_{3} \Pi\left[\alpha(\delta+\mu)(\eta+b)+b^{2} \xi\right]}{b \eta(\eta+b)(\nu+\mu)(\gamma+\delta+\mu)}\right], } \\
a_{3}= & -\eta b(\gamma+\delta+\mu)\left(\mathcal{R}_{0}-1\right) .
\end{aligned}
$$

For $\mathcal{R}_{0}<1$, we have $a_{1}>0, a_{2}>0$ and $a_{3}>0$. It is also direct to show that $a_{1} a_{2}>a_{3}$. Hence, by the Routh-Hurwitz criterion, the real parts of the remaining three eigenvalues are negative. Thus, the disease free equilibrium is locally asymptotically stable for $\mathcal{R}_{0}<1$. When $\mathcal{R}_{0}>1$, the criterion is violated and thus the DFE is unstable.

Proof of Theorem 3.3. We use Kamgang-Sallet Stability Theorem in Ref. 17. Let $X=$ $\left(X_{1}, X_{2}\right), \quad X_{1}=(S, R, T, V) \in \mathbb{R}^{4}$ and $X_{2}=(I, D, P) \in \mathbb{R}^{3}$. Then the system (2.1) can be written as

$$
\begin{aligned}
& \dot{X}_{1}=A_{1}(X)\left(X_{1}-X_{1}^{*}\right)+A_{12}(X) X_{2} \\
& \dot{X}_{2}=A_{2}(X) X_{2}
\end{aligned}
$$

where $X_{1}^{*}=\left(\frac{\Pi}{\nu+\mu}, 0,0, \frac{\nu \Pi}{\mu(\nu+\mu)}\right)$,

$$
A_{1}(X)=\left(\begin{array}{cccc}
-(\nu+\mu) & 0 & 0 & 0 \\
0 & -\mu & 0 & 0 \\
0 & 0 & -\mu & 0 \\
\nu & 0 & 0 & -\mu
\end{array}\right)
$$

$A_{12}(X)$ is

$$
\left(\begin{array}{ccc}
\left(-1-\epsilon \frac{\lambda^{n-1}}{\lambda_{0}^{n}+\lambda^{n}}\right) \frac{\beta_{1} S}{N} & \left(-1-\epsilon \frac{\lambda^{n-1}}{\lambda_{0}^{n}+\lambda^{n}}\right) \beta_{2} S & \left(-1-\epsilon \frac{\lambda^{n-1}}{\lambda_{0}^{n}+\lambda^{n}}\right) \beta_{3} S \\
\gamma & 0 & 0 \\
\left(\frac{\epsilon \lambda^{n-1}}{\lambda_{0}^{n}+\lambda^{n}} S-(1-\theta) T\right) \frac{\beta_{1}}{N} & \left(\frac{\epsilon \lambda^{n-1}}{\lambda_{0}^{n}+\lambda^{n}} S-(1-\theta) T\right) \beta_{2} & \left(\frac{\epsilon \lambda^{n-1}}{\lambda_{0}^{n}+\lambda^{n}} S-(1-\theta) T\right) \beta_{3} \\
0 & 0 & 0
\end{array}\right)
$$

and $A_{2}(X)$ is

$$
\left(\begin{array}{ccc}
\frac{\beta_{1}}{N}[S+(1-\theta) T]-(\gamma+\delta+\mu) & \beta_{2}[S+(1-\theta) T] & \beta_{3}[S+(1-\theta) T] \\
\mu+\delta & -b & 0 \\
\xi & \alpha & -\eta
\end{array}\right)
$$

We show that the five sufficient conditions of Kamgang-Sallet Theorem are satisfied as follows. 
(1) The system (2.1) is a dynamical system on $\Omega$. This is proved in Theorem 3.1.

(2) The equilibrium $X_{1}^{*}$ is GAS for the subsystem $\dot{X}_{1}=A_{1}\left(X_{1}, 0\right)\left(X_{1}-X_{1}^{*}\right)$. This is obvious from the structure of the involved matrix.

(3) The matrix $A_{2}(X)$ is Metzler (i.e., all the off-diagonal elements are nonnegative) and irreducible for any given $X \in \Omega$. This is also obvious.

(4) There exists an upper-bound matrix $\bar{A}_{2}$ for the set

$$
\mathcal{M}=\left\{A_{2}(X): X \in \Omega\right\} .
$$

Indeed, since $\frac{S+(1-\theta) T}{N} \leq 1$,

$$
\bar{A}_{2}=\left(\begin{array}{ccc}
\beta_{1}-(\gamma+\delta+\mu) & \beta_{2} & \frac{\Pi \beta_{3}}{\mu} \\
\mu+\delta & -b & 0 \\
\xi & \alpha & -\eta
\end{array}\right)
$$

is an upper-bound of $\mathcal{M}$.

(5) For $\mathcal{R}_{0} \leq 1$ in $(3.5)$

$$
\alpha\left(\bar{A}_{2}\right)=\max \left\{\operatorname{Re}(\lambda): \lambda \text { eigenvalue of } \bar{A}_{2}\right\} \leq 0 .
$$

Hence, by the Kamgang-Sallet Stability Theorem, the disease-free equilibrium is globally asymptotically stable for $\mathcal{R}_{0} \leq 1$.

Proof of Theorem 3.5. We check the local stability of the endemic equilibrium of the system $(2.1)$ at $\mathcal{R}_{0}=1$. To this end, in the setting of Theorem 4.1 in Ref. 10 where the sign of the numbers $a$ and $b$ below are crucial. We introduce variables $x=\left(x_{1}, x_{2}, x_{3}, x_{4}, x_{5}, x_{6}, x_{7}\right)$ where,

$$
x_{1}=\frac{\Pi}{\mu+\nu}-S, x_{2}=I, x_{3}=R, x_{4}=T, x_{5}=V-\frac{\nu \Pi}{\mu(\mu+\nu)}, x_{6}=D \text { and } x_{7}=P .
$$

Let $\phi=b \eta \mu \beta_{1}+b \eta \Pi(\mu+\delta) \beta_{2}+\beta_{3} \Pi(b \xi+\alpha(\mu+\delta))$ be the bifurcation parameter so that $\mathcal{R}_{0}=\frac{\phi}{b \eta(\nu+\mu)(\gamma+\delta+\mu)}$. Thus $\mathcal{R}_{0}=1$ if and only if $\phi=\phi^{*}=b \eta(\nu+\mu)(\gamma+\delta+\mu)$ and $\mathcal{R}_{0}<1$ if and only if $\phi<\phi^{*}$. Using the above variables, the system (2.1) becomes

$$
\begin{aligned}
\frac{d x_{1}}{d t} & =\left(\epsilon e+\lambda_{1}\right)\left(\frac{\Pi}{\mu+\nu}-x_{1}\right)-(\nu+\mu) x_{1}=: f_{1}, \\
\frac{d x_{2}}{d t} & =\lambda_{1}\left(\frac{\Pi}{\mu+\nu}-x_{1}\right)-(\gamma+\delta+\mu) x_{2}+(1-\theta) \lambda_{1} x_{4}=: f_{2}, \\
\frac{d x_{3}}{d t} & =\gamma x_{2}-\mu x_{3}=: f_{3} \\
\frac{d x_{4}}{d t} & =\epsilon e\left(\frac{\Pi}{\mu+\nu}-x_{1}\right)-(1-\theta) \lambda_{1} x_{4}-\mu x_{4}=: f_{4}, \\
\frac{d x_{5}}{d t} & =-\nu x_{1}-\mu x_{5}=: f_{5} \\
\frac{d x_{6}}{d t} & =(\mu+\delta) x_{2}-b x_{6}=: f_{6} \\
\frac{d x_{7}}{d t} & =\xi x_{2}+\alpha x_{6}-\eta x_{7}=: f_{7},
\end{aligned}
$$

where $N=\frac{\Pi}{\mu}-x_{1}+x_{2}+x_{3}+x_{4}+x_{5}$ and $\lambda_{1}=\frac{\beta_{1} x_{2}}{N}+\beta_{2} x_{6}+\beta_{3} x_{7}$. The diseasefree equilibrium of (A.8) which corresponds to $E_{0}$ is $x^{*}=(0,0,0,0,0,0,0)$. The Jacobian matrix $J$ of the right-side of the system (A.8) at $x^{*}$ denoted by $J\left(x^{*}\right)$ is 


$$
J\left(x^{*}\right)=\left(\begin{array}{ccccccc}
-(\mu+\nu) & \frac{\beta_{1} \mu}{\mu+\nu} & 0 & 0 & 0 & \frac{\beta_{2} \Pi}{\mu+\nu} & \frac{\beta_{3} \Pi}{\mu+\nu} \\
0 & \frac{\beta_{1} \mu}{\mu+\nu}-(\gamma+\delta+\mu) & 0 & 0 & 0 & \frac{\beta_{2} \Pi}{\mu+\nu} & \frac{\beta_{3} \Pi}{\mu+\nu} \\
0 & \gamma & -\mu & 0 & 0 & 0 & 0 \\
0 & 0 & 0 & -\mu & 0 & 0 & 0 \\
-\nu & 0 & 0 & 0 & -\mu & 0 & 0 \\
0 & \mu+\delta & 0 & 0 & 0 & -b & 0 \\
0 & \xi & 0 & 0 & 0 & \alpha & -\eta
\end{array}\right) .
$$

When $\mathcal{R}_{0}=1, J\left(x^{*}\right)$ has a simple zero eigenvalue and all the other eigenvalues have negative real part. This is the setting of Theorem 4.1 in Ref. 10. The right-eigenvector $w=\left(w_{1}, w_{2}, w_{3}, w_{4}, w_{5}, w_{6}, w_{7}\right)$ and the left-eigenvector $v=\left(v_{1}, v_{2}, v_{3}, v_{4}, v_{5}, v_{6}, v_{7}\right)$ associated with the zero eigenvalue such that $w \cdot v=1$ are determined the systems

$$
J\left(x^{*}\right) w=0 . \quad \text { and } \quad v J\left(x^{*}\right)=0 .
$$

Simplification of (A.9) gives

$$
\begin{gathered}
w_{1}=\frac{b \beta_{1} \mu}{(\mu+\nu)(\mu+\delta)}+\frac{\beta_{2} \Pi}{\mu+\nu}+\frac{\beta_{3} \Pi}{(\mu+\nu) \eta(\mu+\delta)}(b \xi+\alpha(\mu+\delta)), \\
w_{2}=\frac{b(\mu+\nu)}{\mu+\delta}, w_{3}=\frac{b \gamma(\mu+\nu)}{\mu(\mu+\delta)}, w_{4}=0, \\
w_{5}=\frac{-\nu}{\mu}\left[\frac{b \beta_{1} \mu}{(\mu+\nu)(\mu+\delta)}+\frac{\beta_{2} \Pi}{\mu+\nu}+\frac{\beta_{3} \Pi}{(\mu+\nu) \eta(\mu+\delta)}(b \xi+\alpha(\mu+\delta)],\right. \\
w_{6}=\mu+\nu \text { and } w_{7}=\frac{(\mu+\nu)(b \xi+\alpha(\mu+\delta))}{\eta(\mu+\delta)} . \\
\left(v_{1}, v_{2}, v_{3}, v_{4}, v_{5}, v_{6}, v_{7}\right)=\left(0, \frac{1}{q}, 0,0,0, \frac{\Pi}{b q(\mu+\nu)}\left(\beta_{2}+\frac{\alpha \beta_{3}}{\eta}\right), \frac{\beta_{3} \Pi}{\eta(\mu+\nu) q}\right),
\end{gathered}
$$

where $q=(\mu+\nu)^{2}\left[\frac{b(\mu+\nu)}{\mu+\delta}+\frac{\beta_{2}}{b}+\frac{\beta_{3} \Pi}{\eta}\left(\frac{\alpha}{b}+\alpha+\frac{b \xi}{\mu+\delta}\right)\right]$. The bifurcation coefficients $a$ and $b$ are

$$
\begin{aligned}
a & =\sum_{k, i, j=1}^{7} v_{k} w_{i} w_{j} \frac{\partial^{2} f_{k}\left(x^{*}, \phi^{*}\right)}{\partial x_{i} \partial x_{j}} \\
& =\sum_{i, j=1}^{7}\left[v_{2} w_{i} w_{j} \frac{\partial^{2} f_{2}\left(x^{*}, \phi^{*}\right)}{\partial x_{i} \partial x_{j}}+v_{6} w_{i} w_{j} \frac{\partial^{2} f_{6}\left(x^{*}, \phi^{*}\right)}{\partial x_{i} \partial x_{j}}+v_{7} w_{i} w_{j} \frac{\partial^{2} f_{7}\left(x^{*}, \phi^{*}\right)}{\partial x_{i} \partial x_{j}}\right] \\
& =v_{2} \sum_{i, j=1}^{7} w_{i} w_{j} \frac{\partial^{2} f_{2}\left(x^{*}, \phi^{*}\right)}{\partial x_{i} \partial x_{j}}, \quad \text { the other terms equal to zero } \\
& =-2 w_{1} v_{2}\left(\beta_{1} w_{2}+\beta_{2} w_{6}+\beta_{3} w_{7}\right) \\
& <0
\end{aligned}
$$

and

$$
\begin{aligned}
b & =\sum_{k, j=1}^{7} v_{k} w_{j} \frac{\partial^{2} f_{k}}{\partial x_{j} \partial \phi}\left(x^{*}, \phi^{*}\right) \\
& =\sum_{j=1}^{7}\left[v_{2} w_{j} \frac{\partial^{2} f_{2}}{\partial x_{j} \partial \phi}\left(x^{*}, \phi^{*}\right)+v_{6} w_{j} \frac{\partial^{2} f_{6}}{\partial x_{j} \partial \phi}\left(x^{*}, \phi^{*}\right)+v_{7} w_{j} \frac{\partial^{2} f_{7}}{\partial x_{j} \partial \phi}\left(x^{*}, \phi^{*}\right)\right] .
\end{aligned}
$$


Further simplification yields

$$
b=\frac{v_{6}}{\eta \beta_{2}+\alpha \beta_{3}}>0 .
$$

Since $a<0$ and $b>0$, the following conclusion holds. When $\phi$ passes through the point $\phi=\phi^{*}$ (or equivalently $\mathcal{R}_{0}$ crosses 1 ) from left to right, the stability of $E_{0}$ changes from globally asymptotic stable (see Theorem 3.2 and 3.3) to unstable and there exists at least one endemic equilibrium which is locally asymptotically stable for $\mathcal{R}_{0}>1$. 